\title{
RAZONES INTRÍNSECAS PARA LA DISCIPLINA EN ESTUDIANTES ADOLESCENTES DE EDUCACIÓN FÍSICA
}

\section{(INTRINSIC REASONS FOR DISCIPLINE IN ADOLESCENT STUDENTS OF PHYSICAL EDUCATION)}

José Antonio Vera Lacárcel

Universidad de Murcia

Juan Antonio Moreno-Murcia

Universidad Miguel Hernández (Elche)

DOI: 10.5944/educXX1.16469

\section{Cómo referenciar este artículo/How to reference this article:}

Vera, J. A., y Moreno, J. A. (2016). Razones intrínsecas para la disciplina en estudiantes adolescentes de Educación Física. Educación XX1, 19(2) 317-335, doi: 10.5944/educXX1.16469

Vera, J. A., \& Moreno, J. A. (2016). Razones intrínsecas para la disciplina en estudiantes adolescentes de Educación Física [Intrinsic reasons for discipline in adolescent students of physical education]. Educación XX1, 19(2) 317-335, doi: 10.5944/educXX1.16469

\section{RESUMEN}

La educación resulta más eficiente cuando las estrategias utilizadas por el profesorado permiten al alumnado tener razones para mantener la disciplina en clase. Este estudio analiza la predicción de las estrategias de disciplina del profesorado, las metas sociales y el apoyo a la autonomía sobre las razones intrínsecas del alumnado para la disciplina en estudiantes adolescentes de educación física. En el estudio participaron 387 estudiantes de Educación Secundaria Obligatoria con edades comprendidas entre los 12 y los 16 años. La información se recogió a través de los cuestionarios de estrategias para mantener la disciplina ( $S S D S)$, metas sociales en educación física ( $S G S-P E)$, apoyo a la autonomía (PASSES) y el factor razones intrínsecas del cuestionario de razones para la disciplina $(R D S)$. El análisis de regresión lineal múltiple mostró que la meta social de responsabilidad, la percepción de estrategias identificadas e intrínsecas y el contexto de apoyo a la autonomía predecían positivamente las razones intrínsecas para mantener la disciplina. Los resultados se discuten en relación al continuo de motivación que presenta la teoría de la autodeterminación. 


\section{PALABRAS CLAVE}

Disciplina; metas sociales; motivación autodeterminada; educación física.

\section{ABSTRACT}

Education is more efficient when teachers use strategies that allow the students have reasons to maintain their own discipline in class. This study analyzes the predictions of the discipline strategies used by the faculty, the social goals and the support for autonomy in the intrinsic reasons of the students for discipline in adolescent students of physical education. A sample of 387 students from secondary education with ages understood between 12 and 16 participated in the study. The information was collected through questionnaires based on the strategies to maintain discipline scale (SSDS), the social goal in physical education (SGS-PE), the autonomy support (PASSES) and the intrinsic reasons factor for the reasons for discipline scale (RDS). Multiple linear regression showed that the social responsibility goal, the identified and intrinsic strategies and the autonomy support positively predicted the intrinsic reasons for discipline positively. The results are discussed in relation to the theory of self-determination.

\section{KEY WORDS}

Discipline; social goals; self motivation; physical education.

\section{INTRODUCCIÓN}

La convivencia en el aula necesita de un clima social positivo para que el profesorado cree unas condiciones adecuadas para la enseñanza (Lewis, 2001). Sin embargo, en la última década, diversos estudios (Ishee, 2004; Koutrouba, Antonopoulou, Tsitsas, y Zenakou, 2009; Maguire, Ball, y Braun, 2010; Uruñuela, 2007) han informado del incremento de la conflictividad, las conductas disruptivas, la desobediencia a las instrucciones del profesorado y el incumplimiento de sanciones en el contexto de enseñanza secundaria, todos ellos, comportamientos relacionados con la falta de interés por la disciplina y la motivación escolar que dejan entrever, entre otros aspectos, la importancia de las estrategias de enseñanza utilizadas para favorecer la disciplina. Estas estrategias deben facilitar el aumento de la participación, sin embargo como plantea Tinning (1997) cuando se llevan a la práctica aparecen problemas en el control del aula que confunden el aprendizaje de la responsabilidad con la obediencia. 
En este sentido, las estrategias utilizadas en educación física pueden favorecer la satisfacción y la motivación en la práctica de actividades físicodeportivas. Éstas deben propiciar coherencia entre la percepción que el alumnado tiene de sí mismo y las actividades propuestas. Cómo indica Fox (1997) la importancia concedida a los diferentes subdominios del autoconcepto físico resulta necesaria para la realización personal. De este modo, la experiencia que el alumnado adquiere en educación física, contribuye a la satisfacción con uno mismo, el rendimiento, el autocontrol, las relaciones sociales y la motivación. Según Rosenberg (1973) la motivación humana es producto de la tendencia universal a mantener, proteger y realizar el autoconcepto.

Desde esta perspectiva, la teoría de la motivación autodeterminada (TAD) (Deci, y Ryan, 1985, 1991, 2000) es un modelo explicativo de la motivación humana que ayuda a comprender la conducta del alumnado en el entorno escolar. Estos autores establecen una clasificación donde la motivación abarca diferentes grados de autodeterminación de la conducta desde la no autodeterminada hasta la autodeterminada, aquella donde el alumnado se convierte en el principal agente causal de su propia vida a través de las decisiones que toma sobre su conducta. El recorrido de una conducta a otra abarca diferentes tipos de motivación desde la desmotivación a la motivación intrínseca. A su vez, cada uno de estos tipos de motivación presenta una regulación por el estudiante que comprende desde la ausencia de regulación en el caso de la desmotivación hasta la regulación interna en la motivación intrínseca. Algunos estudios (Papaioannou, 1998; Spray, 2002) han relacionado los diferentes tipos de motivación que presenta la TAD con la conducta disciplinada en la clase de educación física. En este sentido, los estudiantes pueden presentar diversas razones para mantener la disciplina en función de la regulación que hacen de su conducta. Cuando los estudiantes no son capaces de regular la conducta, presentan razones de desmotivación que se manifiestan en una falta de participación o en la aparición de conductas disruptivas en la clase de educación física. Sin embargo, si la regulación de la conducta la realiza el propio alumnado de forma interna, las razones que presenta son intrínsecas, mantiene la disciplina por la satisfacción que le produce la realización de la actividad.

En esta misma línea, el modelo de motivación extrínseca e intrínseca de Vallerand $(1997,2001)$ ha planteado la existencia de tres niveles motivacionales (situacional, contextual y global), las motivaciones que una persona experimenta en una situación concreta se puedan transferir a otro contexto, como el centro escolar y formar parte de un comportamiento generalizado. El modelo sostiene que existen tres necesidades psicológicas básicas necesarias para el bienestar psicológico (autonomía, competencia y relación con los demás). Ryan y Deci (2000) han señalado que las personas están más 
autodeterminadas cuando tienen satisfechas las necesidades psicológicas básicas. En el caso concreto de la autonomía, la participación en la clase está sujeta a la capacidad de decidir del alumnado. En este sentido, el contexto de apoyo del profesorado a la autonomía ha sido uno de los factores que se ha analizado en educación física. Se concibe este tipo de contexto como el que permite elegir, se opone al control y minimiza la presión en la realización de la tarea. La utilización de climas que apoyan la autonomía en el aula de educación física se ha relacionado con la mejora de la percepción de autonomía (Ntoumanis, 2005; Reinboht, Duda, y Ntoumanis, 2004), la motivación intrínseca (Deci y Ryan, 2000; Gagné, Ryan, y Bargmann, 2003; Prusak, Treasure, Darse, y Pangrazzi, 2004) y la relación con los demás (Balaguer, Castillo, y Duda, 2008; Standage, Duda, y Ntoumanis, 2006). De este modo, la autonomía es una de las necesidades que presenta mayor relación con la motivación autodeterminada (Deci y Ryan, 2000). Así, Bryan y Solmon (2007) sostiene que la satisfacción de esta necesidad psicológica mejora la disciplina.

Aunque el contexto de apoyo a la autonomía aporta información valiosa acerca de la motivación autodeterminada, diversas investigaciones (Moreno, Hernández, y González-Cutre, 2009; Vallerand, 2001) han señalado que existen otros factores sociales relacionados con las metas que persiguen los adolescentes que pueden estar afectando a la motivación. Entre estas metas destaca la importancia que la interacción social tiene en el aula de educación física como condicionante para realizar las tareas. Una de las aportaciones que las metas sociales han realizado al estudio de la motivación ha sido plantear que el deseo de interacción social puede estar afectando al comportamiento de los estudiantes (Anderman, y Anderman, 1999; Guan, McBride, y Xiang, 2006; Moreno, González-Cutre, y Sicilia, 2007). El aula de educación física es un espacio comunicativo donde la interacción social y los factores afectivos adquieren un valor predominante que están en continua negociación con el alumnado. Los estudiantes tienen razones sociales que pueden determinar el comportamiento. En este sentido, Sánchez Arroyo (2009) indicaba que las relaciones sociales en educación física, respecto de otras asignaturas del currículo, son diferentes por la aparición de un mayor dinamismo, libertad de movimientos, mayor complejidad organizativa, por el incremento de las interacciones y los frecuentes cambios de agrupamiento, mayor comunicación verbal y no verbal, interacciones personales y por consiguiente, una gran importancia de la motivación para centrar el comportamiento en la tarea ante las múltiples interacciones recíprocas.

Por todo ello, las metas sociales pueden presentarse como variables relevantes que aporten información acerca de los procesos que conducen a la conducta disciplinada. Las principales metas sociales que se han estudiado 
para explicar la motivación del alumnado son la meta social de responsabilidad y la meta social de relación. La primera hace referencia al interés por respetar las normas sociales y la segunda al deseo de tener relaciones satisfactorias con los iguales (Ryan, Hicks, y Midgley, 1997). Algunos estudios (Cecchini, González, Méndez, Fernández-Río, Contreras, y Romero, 2008; Guan et al., 2006; Papaioannou, Tsigilis, Kosmidou, y Milosis, 2007) han relacionado positivamente las metas sociales con la motivación intrínseca y sus consecuencias, el esfuerzo, la persistencia y el interés por el aprendizaje. En esta misma línea, las meta de responsabilidad predijo positivamente la necesidad de autonomía (Moreno et al., 2009, Moreno-Murcia y Vera, 2011), mientras que la meta de relación no lo hacía cuando la planificación de la autonomía del alumnado no se cedía a través del trabajo cooperativo y la toma de decisiones conjunta (Moreno et al. 2008).

La habilidad para controlar la clase y manejar a los estudiantes es una de las preocupaciones del profesorado (Siedentop, y Tannechill, 1999; Kulinna, Cothran, y Regulaos, 2003) y con esta finalidad utiliza estrategias que mantengan la disciplina. Sin embargo, el estudiante puede percibir que las estrategias utilizadas no permiten la disciplina, por ejemplo, cuando percibe en el profesorado estrategias basadas en la indiferencia a las conductas disruptivas, como falta de interés en cuestiones relacionadas con el cumplimiento de las normas de clase. En segundo lugar, puede percibir la utilización de estrategias introyectadas, razones externas para mantener la disciplina, las estrategias utilizadas para la regulación de la conducta se basan en premiar las conductas adecuadas y censurar las inadecuadas. En tercer lugar, el estudiante puede percibir estrategias identificadas, cuando percibe la preocupación del profesorado para mantener la disciplina pero ésta no va acompañada del disfrute de la actividad física. Por ultimo, encontramos las estrategias intrínsecas, aquellas que hacen sentir al alumnado que actúa bajo su propia autodeterminación más que por las normas impuestas debido a la satisfacción que le produce la participación en la actividad física. Así, de menor a mayor autodeterminación, la percepción de estrategias de indiferencia e introyectadas se ha relacionado con una conducta menos autodeterminada y las estrategias identificadas e intrínsecas con una más autodeterminada (Martínez Galindo, Alonso, Cervelló, y Moreno, 2009; Moreno-Murcia, Martínez Galindo, y Cervelló, 2011; Sarrazin, Vallerand, Guillet, Pelletier, y Cury, 2002; Zounhia, Hatziharistos, y Emmanouel, 2003).

En relación a la percepción que los estudiantes tienen de las estrategias utilizadas por el profesorado para mantener la disciplina, algunas investigaciones (Moreno, Cervelló, y Martínez Galindo, 2007; Psunder, 2005; Zounhia et al., 2003) han encontrado correlaciones positivas entre éstas y las razones del alumnado. Por el contrario, otros estudios (Gotzens, Castelló, 
Genovard, y Badía, 2003; Gutiérrez, López, y Ruiz, 2009) no han encontrado coincidencia entre las estrategias utilizadas y la percepción del estudiante. En este sentido, Gutiérrez, Ruiz, y López (2010) han señalado que en un clima de aula caracterizado por la motivación intrínseca, el grado más elevado de motivación autodeterminada (disfrute, persistencia, percepción de competencia, importancia al esfuerzo e interés) predecía el énfasis del profesorado en razones intrínsecas para mantener la disciplina (estrategias de enseñanza que producen satisfacción y disfrute para el buen comportamiento). Mientras que el énfasis en razones introyectadas (estrategias de enseñanza percibidas por el alumnado como medida de presión externa para la realización de la actividad, premios o castigos) y la indiferencia del profesorado, predecían la motivación intrínseca negativamente. Estos resultados apoyan la idea de que la mejora de la disciplina está estrechamente vinculada con el diseño de un contexto de aula basado en la motivación autodeterminada. Cuanto más autodeterminada es la conducta del alumnado más acorde está con un sistema de valores que informa de un mayor bienestar psicológico (Deci y Ryan, 1991).

El objetivo de este estudio ha sido comprobar la capacidad de predicción de las metas sociales, el apoyo a la autonomía y la percepción de las estrategias de disciplina utilizadas por el profesorado sobre las razones intrínsecas para la disciplina en estudiantes adolescentes en clases de educación física. Según los estudios presentados, pretendemos comprobar que las metas sociales, el apoyo a la autonomía y las estrategias basadas en razones identificadas e intrínsecas predecirían positivamente las razones intrínsecas del alumnado y que las estrategias basadas en razones introyectadas y en la indiferencia del profesorado las predecirán negativamente.

\section{MATERIAL Y MÉTODO}

\section{Participantes}

La muestra estuvo compuesta por 387 estudiantes españoles, 199 hombres y 188 mujeres, con una media de edad de 14.63 años $(D T=2.03)$ procedentes de siete centros públicos (55 estudiantes aproximadamente por centro) ubicados en un entorno urbano de una gran ciudad española y seleccionados intencionadamente.

\section{Instrumentos}

Metas sociales. Se empleó la Social Goal Scale-Physical Education (SGS-PE) de Guan et al. (2006), validada al contexto español por Moreno et 
al. (2007). Esta escala está compuesta por un total de 11 reactivos agrupados en dos factores: meta de relación (e. g. «Me gustaría tener un amigo/a en el que poder confiar») y meta de responsabilidad (e. g. «Es importante para mí seguir las reglas de clase»). La sentencia previa fue «En mis clases de educación física...» y las preguntas se respondían mediante una escala tipo Likert que oscilaba entre 1 (totalmente en desacuerdo) y 7 (totalmente de acuerdo). En el presente estudio se obtuvieron valores alfa de Cronbach de .78 para la meta de relación y .75 para la meta de responsabilidad.

Estrategias para mantener la disciplina. Se utilizó la versión en español (Moreno, Cervelló, Martínez Galindo, y Ruiz, 2008) del cuestionario Strategies to Sustain Discipline Scales (Papaioannou, 1998) para medir las estrategias percibidas por el alumnado y mostradas por el docente para mantener la disciplina en la clase. El cuestionario está formado por 27 ítems agrupados en cuatro factores: Estrategias identificadas, basadas en razones de preocupación y responsabilidad (e. g. «El/la profesor/a nos ayuda a ser responsables de nuestro progreso»), estrategias intrínsecas, basadas en razones para actuar bajo la propia autodeterminación (e. g. «El/la profesor/a atrae nuestra atención y hace las clases más interesantes»), estrategias introyectadas, basadas en gratificar las conductas apropiadas y castigar las inapropiadas (e. g. «El/la profesor/a nos hace sentirnos mal con nosotros mismos cuando somos indisciplinados») y estrategias de indiferencia, basadas en la falta de interés por el mantenimiento del orden (e. g. «El/la profesor/a no se interesa por la disciplina en clase»). Las respuestas a dicho cuestionario eran cerradas y respondían a una escala tipo Likert que iba de 0 , valor que correspondía a totalmente en desacuerdo, a 10 que indicaba que el alumnado estaba totalmente de acuerdo con lo que se le planteaba. Los ítems estaban precedidos por la frase «El/la profesor/a de educación física...». Se obtuvieron valores alfa de Cronbach de .86, .90, .72. y .76, respectivamente.

Apoyo a la autonomía. Se empleó la validación al contexto español (Moreno, Parra, y González-Cutre, 2008) de la Perceived Autonomy Support Scale for Exercise Settings (Hagger, Chatzisarantis, Hein, Pihu, Soós, y Karsai, 2007). Esta escala está compuesta de 12 ítems (e. g. "Soy capaz de compartir mis experiencias de ejercicio físico o deportivo con mi profesor/a», «Confío en el consejo que el profesor/a me da sobre el ejercicio físico o deportivo que hago en mi tiempo libre»), agrupados en un solo factor (apoyo a la autonomía por parte del docente). La sentencia previa fue «En mis clases de educación física...» La escala se responde mediante una escala tipo Likert de 1 (totalmente en desacuerdo) a 7 (totalmente de acuerdo). En este estudio el valor alfa de Cronbach fue de .90 .

Razones de disciplina. Se empleó el factor «razones intrínsecas para la disciplina» del Cuestionario Reasons for Discipline (RDS) de Papaioannou, 
1998, validada al contexto español por Moreno et al. (2008). El factor está formado por 4 ítems (e. g. «Disfruto de las clases»). Las respuestas a dicho cuestionario eran cerradas y respondían a una escala tipo Likert que iba de 0 , valor que correspondía a totalmente en desacuerdo, a 10 que indicaba que el alumnado estaba totalmente de acuerdo con lo que se le planteaba. Los ítems estaban precedidos por la frase «En clase de educación física...». En el presente estudio se obtuvieron valores alfa de Cronbach de .84.

\section{Procedimiento}

Para la recogida de la información se estableció contacto con el profesorado de educación secundaria para pedirles su colaboración en el estudio y se les instruyó debidamente. Se solicitó al alumnado una autorización por escrito de sus padres por ser menores de edad, dando el consentimiento la totalidad de los mismos. La administración de los factores seleccionados de cada escala se realizó en el mismo orden en cada uno de los grupos y en presencia del investigador para explicar el objetivo y estructura de la investigación, así como la forma de cumplimentarlas. Se informó del anonimato de las respuestas. Durante dicho proceso el investigador solventó aquellas dudas que surgieron al respecto. La duración aproximada de la cumplimentación de los cuestionarios fue de 15 minutos y ésta se realizó de forma voluntaria.

\section{Análisis de datos}

Se calcularon los estadísticos descriptivos y se realizó un análisis de correlaciones bivariadas con todas las variables. También se realizó un Anova de un factor para analizar las posibles diferencias en función del género del alumnado y cada una de los factores de estudio. Para comprobar el poder de predicción de las estrategias de disciplina, las metas sociales y el apoyo a la autonomía sobre las razones intrínsecas para la disciplina se llevó a cabo un análisis de regresión lineal por pasos con el paquete estadístico SPSS 19.0.

\section{RESULTADOS}

\section{Análisis descriptivo y de correlación}

En los estadísticos descriptivos (Tabla 1) cabe destacar la percepción que el alumnado mostró en la percepción de las «razones intrínsecas» para la disciplina $(M=6.14)$, las «estrategias identificadas» utilizadas por el profesorado $(M=6.04)$, así como la importancia de la «meta social de relación» $(M=6.00)$, las «estrategias intrínsecas» $(M=5.42)$, la «meta social de res- 
ponsabilidad» $(M=4.83)$ y el «apoyo a la autonomía» $(M=4.43)$. Por otro lado, es también destacable la baja percepción que el alumnado mostró en las «estrategias introyectadas» $(M=4.35)$ y las «estrategias de indiferencia» $(M=3.83)$. En relación a las desviaciones típicas destacaba la uniformidad y poca variabilidad de los factores que forman las metas sociales, las estrategias y las razones intrínsecas.

El análisis de correlación entre las variables del estudio (Tabla 1) mostraron que las correlaciones más destacadas son las que relacionan positiva y significativamente las «razones intrínsecas» del alumnado para mantener la disciplina con la «meta social de responsabilidad» $(r=.49)$, la «meta de relación» $(r=.42)$, el «apoyo a la autonomía» $(r=.43)$, las «estrategias identificadas» $(r=.41)$ y las «estrategias intrínsecas» $(r=.55)$. Mientras que esta relación fue negativa y significativa para las «estrategias introyectadas» $(r=-.14)$ y las "estrategias de indiferencia» $(r=-.17)$. Por otro lado, cuando el alumnado percibía que el profesorado apoyaba la autonomía en la práctica de actividades físico-deportivas, esto correlacionaba con la percepción de «estrategias identificadas $(r=.61)$ y con las «estrategias intrínsecas» $(r=.67)$. La percepción de «estrategias identificadas» $\mathrm{y}$ «estrategias intrínsecas» en el alumnado correlacionaba negativa y significativamente con las «estrategias de indiferencia» $(r=-.11)$ y $(r=-.15)$. Es decir, el alumnado que percibe estrategias de disciplina más autodeterminadas no percibía estrategias de indiferencia en el profesorado.

Tabla 1.

Estadísticos descriptivos y correlaciones de todas las variables

\begin{tabular}{l|c|c|c|c|c|c|c|c|c|c}
\hline & $M$ & $D T$ & $\mathbf{1}$ & $\mathbf{2}$ & $\mathbf{3}$ & $\mathbf{4}$ & $\mathbf{5}$ & $\mathbf{6}$ & $\mathbf{7}$ & $\mathbf{8}$ \\
\hline 1. Responsabilidad & 4.83 & .81 & - & $.49 * *$ & $.23^{* *}$ & $.22^{* *}$ & $.17^{* *}$ & $.20^{* *}$ & $-.13^{* *}$ & $.49^{* *}$ \\
2. Relación & 6.00 & .98 & - & - & $.11^{*}$ & $.16^{* *}$ & $.18^{* *}$ & $.11^{*}$ & $-.10^{*}$ & $.42^{* *}$ \\
3. Apoyo a la autonomía & 4.43 & 1.58 & & - & - & $.61^{* *}$ & $.67^{* *}$ & $.38^{* *}$ & $.14^{* *}$ & $.43^{* *}$ \\
4. E. identificadas & 6.04 & 2.15 & - & - & - & - & $.48^{* *}$ & $.24 * *$ & $-.11^{*}$ & $.41^{* *}$ \\
5. E. intrínsecas & 5.42 & 2.51 & - & - & - & - & - & $.23^{* *}$ & $-.15^{* *}$ & $.55^{* *}$ \\
6. E. introyectadas & 4.35 & 2.19 & - & - & - & - & - & - & $.15^{* *}$ & $-.14^{* *}$ \\
7. E. indiferencia & 3.83 & 2.45 & - & - & - & - & - & - & - & $-.17^{* *}$ \\
8. R. intrínsecas & 6.14 & 2.50 & - & - & - & - & - & - & - & - \\
\hline
\end{tabular}

Nota: * $\mathrm{p}<.05 ; * * \mathrm{p}<.01 ; E=$ Estrategias, $R=$ Razones

\section{Diferencias según el género del alumnado}

El Anova (Tabla 2) mostró que en el género del alumnado existían diferencias en las estrategias identificadas, las estrategias intrínsecas y las 
razones intrínsecas para la disciplina, no encontrándose para el resto de variables dependientes. En relación a las diferencias, los alumnos mostraron una mayor percepción de las estrategias identificadas $(F=6.22, p=.013) \mathrm{e}$ intrínsecas $(F=5.19, p=.023)$ utilizadas por el profesorado y de las razones intrínsecas $(F=4.43, p=.036)$ para la disciplina que las alumnas.

Tabla 2.

Resultados diferenciados según el género del alumnado

\begin{tabular}{llccc}
\hline & Chicos & Chicas & $F$ & $p$ \\
\hline M. Responsabilidad & 4.76 & 4.90 & 2.81 & .093 \\
M. Relación & 5.90 & 6.09 & 3.40 & .066 \\
Apoyo a la autonomía & 4.57 & 4.27 & 3.54 & .060 \\
E. identificadas & 6.31 & 5.76 & $6.22^{*}$ & .013 \\
E. Intrínsecas & 5.71 & 5.12 & $5.19^{*}$ & .023 \\
E. Introyectadas & 4.31 & 4.39 & .106 & .745 \\
E. Indiferencia & 3.59 & 3.88 & 1.31 & .253 \\
R. Intrínsecas & 6.41 & 5.86 & $4.43^{*}$ & .036 \\
\hline
\end{tabular}

Nota: * $\mathrm{p}<.05 ; * * \mathrm{p}<.01 ; E=$ Estrategias, $\mathrm{R}=$ Razones

\section{Análisis de regresión lineal por pasos}

Se realizó un análisis de regresión lineal con el método de introducir con tres pasos considerando como variables independientes las metas sociales, el apoyo a la autonomía y la percepción de las estrategias de disciplina del profesorado sobre las razones intrínsecas del alumnado para la disciplina. En la introducción de las variables se comprobó el diagnóstico de colinealidad resultando variables no-colineales con un índice de condición que no supera el valor de 15 (Belsley, 1991). El orden en la introducción de las variables se realizó siguiendo las consideraciones del modelo jerárquico de Valerand (1997, 2001). De este modo, en el primer paso, se introdujeron las metas sociales, en el segundo, el contexto de apoyo a la autonomía y en el tercero, la percepción de las estrategias utilizadas por el profesorado para mantener la disciplina.

El análisis de regresión (Tabla 3) mostró tres modelos significativos para las razones intrínsecas. El coeficiente global de determinación $\left(R^{2}=.57\right)$ indicó que las variables independientes consideradas en el estudio contribuyen en un 57\% a explicar la varianza de las «razones intrínsecas» para 
la disciplina. En el primer paso, el coeficiente de determinación $\left(R^{2}=.10\right)$ hizo que "la meta de responsabilidad» $(\beta=.22)$ predijera positivamente las «razones intrínsecas» como variable que más contribuye a la disciplina del alumnado, explicando el 10\% de la varianza. En el segundo paso se añadió el «apoyo a la autonomía», produciéndose un incremento del coeficiente de determinación $\left(R^{2}=.37\right)$, en este caso, la varianza explicada aumenta un $27 \%$, siendo las variables que muestran la predicción positiva y significativa, la «meta de responsabilidad» $(\beta=.16)$ y «el apoyo a la autonomía» $(\beta=.35)$. En el tercer paso se incluyeron la percepción de las estrategias para mantener la disciplina, «estrategias identificadas», «estrategias intrínsecas», «estrategias introyectadas» $\mathrm{y}$ «estrategias de indiferencia», la varianza explicada aumentó un $47 \%$ en relación al paso primero y un $20 \%$ con respecto al segundo. Las variables con mayor capacidad predictiva fueron la «meta social de responsabilidad» $(\beta=.10)$, el «apoyo a la autonomía» $(\beta=.14)$, las «estrategias identificadas» $(\beta=.22)$ y las «estrategias intrínsecas» $(\beta=.44)$.

Tabla 3.

Resumen del Análisis de Regresión Múltiple de las razones Intrínsecas del Alumnado Para Mantener la Disciplina Según las Metas Sociales, el Apoyo a la Autonomía y la Percepción de las Estrategias del Profesorado

\begin{tabular}{lccccc}
\hline \multicolumn{1}{c}{ Razones Intrínsecas } & B & $\begin{array}{c}\text { Error } \\
\text { típico }\end{array}$ & $\boldsymbol{\beta}$ & $\boldsymbol{t}$ & $\boldsymbol{R}^{\mathbf{2}}$ \\
\hline Paso 1 & & & & & $10 * *$ \\
Responsabilidad & .72 & .16 & .22 & $4.38 * *$ & \\
Relación & -.02 & .15 & -.00 & -.14 & \\
Paso 2 & & & & & $37 * *$ \\
Responsabilidad & .50 & .13 & .16 & $3.75 * *$ & \\
Relación & .04 & .12 & .01 & .38 & \\
Autonomía & .53 & .08 & .35 & $6.59 * *$ & \\
Paso 3 & & & & & $57 * *$ \\
Responsabilidad & .32 & .11 & .10 & $2.73 * *$ & \\
Relación & .08 & .08 & .14 & .80 & \\
Autonomía & .22 & .08 & .14 & $2.75 * *$ & \\
E. identificadas & .25 & .07 & .22 & $3.64 * *$ & \\
E. intrínsecas & .44 & .06 & .44 & $6.83 * *$ & \\
E. introyectadas & -.04 & .04 & -.03 & -.85 & \\
E. Indiferencia & -.03 & .04 & -.04 & -.98 & \\
\hline
\end{tabular}

Nota: $* \mathrm{p}<.05 ; * * \mathrm{p}<.01 ; E=$ Estrategias 


\section{DISCUSIÓN}

El objetivo de este estudio ha sido comprobar la capacidad de predicción de las metas sociales, el apoyo a la autonomía y la percepción de las estrategias de disciplina utilizadas por el profesorado, sobre las razones intrínsecas para mantener la disciplina en estudiantes adolescentes de educación física. Estas razones se estudian en relación a los diferentes niveles de motivación que señala la teoría de la autodeterminación (Deci y Ryan, 1985) y que pueden utilizarse según diversos autores (Papaioannou, 1998; Spray, 2002) para explicar las estrategias de disciplina utilizadas por el profesorado en educación física. El análisis de regresión lineal múltiple mostró que la meta social de responsabilidad, el apoyo a la autonomía y las estrategias identificadas e intrínsecas predecían positivamente las razones intrínsecas para la disciplina. Del mismo modo, el análisis de correlaciones bivariadas mostró una relación positiva y significativa entre la metas sociales, el apoyo a la autonomía, las estrategias identificadas e intrínsecas y las razones intrínsecas y una relación negativa y significativa entre las estrategias introyectadas y de indiferencia y las razones intrínsecas para la disciplina. Estudios anteriores (Moreno et al., 2008) mostraron una relación positiva entre las metas sociales y el apoyo a la autonomía y entre la meta social de responsabilidad, la necesidad psicológica de autonomía y la motivación intrínseca (Moreno et al., 2009; Moreno y Vera, 2011). Del mismo modo, las estrategias identificadas e intrínsecas se han relacionado positivamente con las razones intrínsecas, mientras las estrategias introyectadas y la indiferencia lo hacían negativamente (Gutiérrez et al., 2009: Moreno et al., 2009). Por tanto, parece que cuando el profesorado cede responsabilidad para que el alumnado actúe con autonomía, éste percibe que las estrategias utilizadas para mantener la disciplina guardan relación con las estrategias identificadas, percibe la preocupación del profesorado para mantener la disciplina. Igualmente, percibe el énfasis en estrategias intrínsecas cuando se compromete a mantener la disciplina porque el clima de aula produce satisfacción con la actividad que realiza. En este sentido, estimular las razones intrínsecas para la disciplina se consigue con la creación de ambientes de enseñanza donde la responsabilidad y la autonomía cedida favorezcan la satisfacción. Sin embargo, las diferencias encontradas en función del género del alumnado apuntan a que los chicos perciben con más fuerza las estrategias identificadas e intrínsecas y las razones intrínsecas para la disciplina que las chicas, lo que sitúa a éstos con mayores razones autodeterminadas para disfrutar de la actividad y por tanto mantener la disciplina en educación física. Estos resultados han sido avalados por diferentes investigaciones (Moreno, Alonso, Martínez Galindo, y Cervelló, 2005; Cervelló, Del Villar, Jiménez, Ramos, y Blázquez, 2003) que manifiestan que la percepción de un clima motivador se relaciona con la igualdad de trato y los comportamientos de disciplina. Parece que pue- 
den existir distintos niveles de gradación en el tipo de motivación percibida según el género que condiciona las razones para la disciplina.

A diferencia de lo planteado en la hipótesis y de los resultados obtenidos por otras investigaciones (Guan et al., 2006; Moreno et al., 2009; Papaioannou et al., 2007) la meta social de relación no predijo las razones intrínsecas para la disciplina. Cabe plantear que si los estudiantes no perciben la necesidad del trabajo cooperativo porque la interacción grupal no conduce hacia una interdependencia positiva, es decir, el progreso de los miembros del grupo, resulte verosímil que en este caso la meta de relación no prediga las razones intrínsecas para la disciplina.

Según el modelo de Vallerand $(1997,2001)$ la necesidad que los estudiantes tienen de relacionarse les conduce hacía la motivación intrínseca lo que implica razones más autodeterminadas para mantener la disciplina en clase. Sin embargo, el estudio no parece confirmar esta parte de la hipótesis, siendo la meta de responsabilidad, el apoyo a la autonomía y las estrategias identificadas e intrínsecas las que aparecen como predictoras de las razones intrínsecas para la disciplina. De este modo, el deseo de respetar las reglas sociales del grupo puede percibirse como un proceso regulador del orden social que no es intrínsecamente atrayente. Es el caso del alumnado que percibe que gana prestigio en el grupo cuando desobedece las instrucciones del profesorado pues este es el modo de operar del grupo-clase que le permite obtener reconocimiento social, hasta aquel que entiende que ser responsable son las razones que mueven el comportamiento del grupo.

Por otro lado, la meta de responsabilidad viene a predecir en menor medida las razones intrínsecas para la disciplina que el apoyo a la autonomía y las estrategias identificadas e intrínsecas. Parece que conforme se avanza en el continuo de autodeterminación donde varias identificaciones son asimiladas, ordenadas jerárquicamente y puestas en congruencia con otros valores (Ryan y Deci, 2000) se observa que la importancia de las metas sociales como desencadenantes de conductas disciplinadas va disminuyendo.

En esta línea, el apoyo a la autonomía es otro desencadenante social que predice las razones intrínsecas (Bryan y Solmon, 2007; Taylor y Ntoumanis, 2007). Cuando el docente hace sentirse más autónomo al alumnado, la satisfacción de esta necesidad psicológica favorece que se encuentre más motivado (Moreno et al., 2009). Por tanto, cuando el alumnado percibe la preocupación del profesorado por incidir en el mantenimiento de la disciplina, la utilización de un estilo de enseñanza basado en la cesión de responsabilidad donde se planifica la autonomía basada en el disfrute de la 
práctica físico-deportiva, el autocontrol del alumnado se manifiesta por la satisfacción que el comportamiento tiene en sí mismo.

Estos resultados vienen a explicar la importancia que el énfasis en estrategias identificadas, aquellas que favorecen la regulación del comportamiento disciplinado pero la decisión final viene dada por una serie de beneficios externos y en estrategias intrínsecas, aquellas en las que el alumnado se compromete a mantener la disciplina porque forma parte de su comportamiento, tienen, debido a que predicen las razones intrínsecas del alumnado, resultados que se han encontrado en diversas investigaciones (Gutiérrez et al., 2009; Jiménez, Cervelló, García, Santos-Rosa, y Del Villar, 2006; Spray, 2002). Por todo ello, las estrategias para mejorar la disciplina en el aula de educación física pasan por dar a conocer al alumnado las reglas de las que tiene que responsabilizarse, planificar la autonomía de la práctica, mostrar preocupación por la aceptación de responsabilidad y ofrecer tareas que favorezcan la satisfacción y la diversión.

Por último, destacar que promover razones intrínsecas en el alumnado para mantener la disciplina está condicionado por la presencia de diversos factores que interrelacionan conjuntamente y que podrían ser motivo de valoración del profesorado. Así, el énfasis en estrategias basadas en razones identificadas, muestra un docente que se preocupa y le importa el desarrollo personal del alumnado, las basadas en razones intrínsecas permiten que la autorregulación de la conducta se lleve a cabo por la satisfacción del aprendizaje, la meta de responsabilidad posibilita respetar las reglas sociales que el grupo se da y que deben basarse en la responsabilidad de las actividades físicas encomendadas. Mientras, el apoyo a la autonomía, favorece la participación desde la elección en la toma de decisiones.

Este trabajo no establece relaciones causales debido a que es un estudio correlacional. Sin embargo, las relaciones que las metas sociales, el apoyo a la autonomía y las estrategias del profesorado muestran representan un avance en el estudio de la disciplina a través de la autodeterminación. Las limitaciones del estudio pueden abordarse en futuros estudios experimentales donde considerar la aplicación de estrategias intrínsecas basadas en técnicas de aprendizaje cooperativo para comprobar los efectos en las razones del alumnado para mantener la disciplina. Del mismo modo, estudios cualitativos deberían aclarar que barreras presenta el profesorado para aplicar estrategias de enseñanza que promuevan la disciplina en la clase de educación física. No obstante, el estudio facilita información para la mejora del comportamiento del alumnado en la clase de educación física dada la dificultad que la enseñanza presenta en el nuevo contexto escolar. 


\section{REFERENCIAS BIBLIOGRÁFICAS}

Anderman, L. H. \& Anderman, E. M. (1999). Social predictors of changes in students' achievement goal orientations. Contemporary Educational Psychology, 25, 21-37.

Balaguer, I., Castillo, I., y Duda, J. L. (2008). Apoyo a la autonomía, satisfacción de las necesidades, motivación y bienestar en deportistas de competición: Un análisis de la Teoría de la Autodeterminación. Revista de Psicología del Deporte, 17(1), 123-139.

Belsley, D. A. (1991). Conditioning diagnostics: Collinearity and Weak Data in Regression. New York: John Wiley \& Son.

Bryan, C. L., y Solmon, M. A. (2007). Self-determination in physical education: designing class environments to promote active lifestyles. Journal of Teaching in Physical Education, 26, 260-278.

Cecchini, J. A., y Peña, J. V. (2005). Estudio experimental de las repercusiones del clima motivacional sobre la orientación de meta, la motivación intrínseca y los comportamientos disruptivos en las clases de Educación Física. Revista Bordón. 57(5), 597-608.

Cecchini, J. A., González, C., Méndez, A., Fernández-Río, J., Contreras, O. y Romero, S. (2008). Metas sociales y de logro, persistencia-esfuerzo e intenciones de práctica deportiva en el alumnado de Educación Física. Psicothema, 20, 260-265.

Cervelló, E., Del Villar, F., Jiménez, R., Ramos, L., y Blázquez, F. (2003). Clima motivacional en el aula, criterios de éxito de los discentes y percepción de igualdad de trato en función del género en las clases de Educación Física. En- señanza: Anuario Interuniversitario de Educación, 21, 379-395.

Deci, E. L., y Ryan, R. M. (1985). Intrinsic motivation and selfdetermination in human behavior. Nueva York: Plenum.

Deci, E. L., y Ryan, R. M. (1991). A motivational approach to self: Integration in personality. En R. Dienstbier (Ed.), Nebraska symposium on motivation: Vol. 38. Perspectives on motivation (pp. 237-288). Lincoln, NE: University of Nebraska Press.

Deci, E. L. \& Ryan, R. M. (2000). The "what» and "why» of goal pursuits: Human needs and the self-determination of behaviour. Psychological Inquiry, 11, 227-268.

Fox, K. R. (1997). The physical self and processes in self-esteem development. En K. R. Fox (Ed.), The physical self from motivation to well-being (pp. 111140). Champaing, IL: Human Kinetics.

Gagné, M., Ryan, R. M. \& Bargmann, K. (2003). Autonomy support and need satisfaction in the motivation and wellbeing of gymnasts. Journal of Applied Sport Psychology, 15, 372-390.

Gotzens, C., Castelló, A., Genovard, C., y Badía, M. (2003). Percepciones de profesores y alumnos de E. S. O, sobre la disciplina en el aula. Psicothema, 15(3), 362-368.

Guan, J., McBride, R. E., y Xiang, P. (2006). Reliability and validity evidence for the Social Goal Scale-Physical Education (SGS-PE) in high school settings. Journal of Teaching in Physical Education, 25, 226-238.

Gutiérrez, M., López, E., y Ruiz, L. M. (2009). Estrategias para mantener la disciplina en las clases de educación física: validación de su medida y aná- 
lisis de la concordancia entre las percepciones de los profesores y las de sus alumnos. Revista Mexicana de Psicología, 26(2), 203-212.

Gutiérrez, M., Ruiz, L. M., y López, E. (2010). Perceptions of motivational climate and teachers' strategies to sustain discipline as predictors of intrinsic motivation in physical education. Spanish Journal of Psychology, 13(2), 597-608.

Hagger, M. S., Chatzisarantis, N. L. D., Hein, V., Pihu, M., Soós, I., y Karsai, I. (2007). The perceived autonomy support scale for exercise settings (PASSES): Development, validity and cross-cultural invariance in young people. Psychology of Sport and Exercise, 8, 632-653.

Ishee, J H. (2004). Perceptions of Misbehaviour in middle school in physical education. Journal of Physical Education, Recreation and Dance, 75, 9.

Jiménez, R., Cervelló, E. M., García, T., Santos-Rosa, F. J., y Del Villar, F. (2006). Relaciones entre las metas de logro, la percepción del clima motivacional, la valoración de la educación física, la práctica deportiva extraescolar y el consumo de drogas en estudiantes de educación física. Revista Mexicana de Psicología, 23, 253-26.

Kulinna, P., Cothran, D., y Regualos, R. (2003). Development of an instrument to measure student disruptive behaviour. Measurement in Physical Education and Exercise Science, 7, 1, 25-41.

Koutrouba, K., Antonopoulou, E., Tsitsas, G., y Zenakou, E. (2009). An investigation of Greek teachers' views on parental involvement in education. School Psychology International, 30(3), 311-318.

Lewis, R. (2001). Classroom discipline student responsibility: the students' view. Teaching and Teacher Education, 17, 307-319.

Maguire, M., Ball, S., y Braun, A. (2010). Behaviour classroom management and student «control»: enacting policy in the English secondary school. International Studies in Sociology of Education, 20(2), 153-170.

Martínez Galindo, C., Alonso, N., Cervelló, E., y Moreno, J. A. (2009). Perfiles motivacionales y disciplina en clases de educación física. Diferencias según las razones del alumnado para ser disciplinado y la percepción de trato generado por el profesor en el aula. Cultura y Educación, 13, 331-343.

Moreno, J. A., Alonso, N., Martínez Galindo, C., y Cervelló, E. (2005). Motivación, disciplina, coeducación y estado de flow en Educación Física: diferencias según la satisfacción, la práctica deportiva y la frecuencia de práctica. Cuadernos de Psicología del Deporte, 5, 1-2, 225-243.

Moreno, J. A., Cervelló, E., y Martínez Galindo, C. (2007). Perception of discipline according to gender, type of school, sport activity and interest in physical education in Spanish students. International Journal of Applied Sports Sciences, 19(2), 35-49.

Moreno, J. A., González-Cutre, D., y Sicilia, A. (2007). Metas sociales en las clases de educación física. Análisis y Modificación de Conducta, 33, 351-368.

Moreno, J. A., Parra, N., y González-Cutre, D. (2008). Influencia del apoyo a la autonomía, las metas sociales y la relación con los demás sobre la desmotivación en educación física. Psicothema, 20, 636-641.

Moreno, J. A., Cervelló, E., Martínez Galindo, C., y Ruiz, L. M. (2008). Preliminary construct validation study of the reason for discipline and strategies to 
sustain discipline scales in Spanish physical education. International journal of Hispanic psychology, 1(1), 85-97.

Moreno, J. A., Hernández-Paños, A., y González-Cutre, D. (2009). Complementando la teoría de la autodeterminación con las metas sociales. Un estudio sobre la diversión en educación física. Revista Mexicana de Psicología, 26(2), 213-222.

Moreno-Murcia, J. A., y Vera, J. A. (2011). Modelo causal de la satisfacción con la vida en adolescentes de educación física. Revista de Psicodidáctica, 16(2), 367-380.

Moreno-Murcia, J. A., Martínez Galindo, C., y Cervelló, E. (2011). Relación predictiva entre la percepción del alumnado de las estrategias de disciplina del profesor y la percepción de igualdad de trato-discriminación en las clases de educación física. Revista de Educación, 355(2), 381-403.

Ntoumanis, N. (2005). A prospective study of participation in optional school physical education using a self-determination theory framework. Journal of Educational Psychology, 97, 444-453.

Papioannou, A. (1998). Goal perspectives, reasons for being disciplined and self-reported discipline in physical education lessons. Journal of Teaching in Physical Education, 17, 421-441.

Papaioannou, A. G., Tsigilis, N., Kosmidou, E. \& Milosis, D. (2007). Measuring perceived motivational climate in physical education. Journal of Teaching in Physical Education, 26, 236-259.

Prusak, K. A., Treasure, D. C., Darst, P. W. \& Pangrazi, R. P. (2004). The effects of choice on the motivation of adolescent girls in physical education. Journal of Teaching in Physical Education, 23, 19-29.
Psunder, M. (2005). How effective is school discipline in preparing students to become responsible citizens? Slovenian teachers' and students' views. Teaching and Teacher Education, 21, 273-286.

Reinboht, M., Duda, J. L., y Ntoumanis, N. (2004). Dimensions of coaching behavior, need satisfaction, and the psychological and physical welfare of young athletes. Motivation and Emotion, 28(3), 297-313.

Rosenberg, M. (1979). Conceiving the self. New York: Basic Books.

Ryan, A. M., Hicks, L., y Midgley, C. (1997). Social goals, academic goals, and avoiding seeking help in the classroom. Journal of Early Adolescence, 17, 152-171.

Ryan, R. M., y Deci, E. L. (2000). Selfdetermination theory and the facilitation of intrinsic motivation, social development and well-being. American Psychologist, 55, 68-78.

Sánchez, F. J. (2009). Análisis del clima de aula en educación física. Un estudio de casos [Tesis Doctoral]. Universidad de Málaga, Málaga.

Sarrazin, P., Vallerand, R., Guillet, E., Pelletier, L. \& Cury, F. (2002). Motivation in dropout in female hyballers: a 21-month prospective study. European Journal of Social Psychology, 32, 395, 418.

Siedentop, D., y Tannehill, D. (1999). Developing teaching skills in physical education. Palo Alto, CA: Mayfield.

Spray, C. M. (2002). Motivational climate and perceived strategies to sustain pupils' discipline in physical education. European Physical Education Review, 8(1), 5-20.

Standage, M., Duda, J. L., y Ntoumanis, N. (2006). Students' motivational 
processes and their relationship to teacher ratings in school physical education: A self-determination theory approach. Research Quarterly for Exercise and Sport, 77, 100-110.

Taylor, I. M., y Ntoumanis, N. (2007). Teacher motivational strategies and student self-determination in physical education. Journal of Educational Psychology, 99, 747-760.

Tinning, R. (1996). Discursos que orientan el campo del movimiento humano y el problema de la formación de la formación del profesorado. Revista de Educación, 311, 123-124.

Uruñuela, P. M. (2007). Convivencia y disrupción en las aulas. Cuadernos de Pedagogía, 364,102-107.
Vallerand, R. J. (1997). Toward a Hierarchical Model of Intrinsic and Extrinsic Motivation. M. P. Zanna (Ed.), Advances in Experimental Social Psychology (vol. 29, pp. 271-360). NY: Academic Press.

Vallerand, R. J. (2001). A hierarchical model of intrinsic and extrinsic motivation in sport and exercise. En G. C. Roberts (Ed.), Advances in motivation in sport and exercise (pp. 263-319). Champaign, IL: Human Kinetics.

Zounhia, K., Hatziharistos, D. \& Emmanouel, K. (2003) Greek secondary school pupils' perceived reasons for behaving appropriately and perceived teachers' strategies to maintain discipline. Educational Review, 55(3), 289-303. 


\section{PERFIL ACADÉMICO Y PROFESIONAL DE LOS AUTORES}

José Antonio Vera Lacárcel. Doctor en Educación Física. Profesor Asociado de la Universidad de Murcia. Actualmente compagina su labor docente en un centro de enseñanza secundaria con la docencia y la investigación en el Departamento de Expresión Plástica, Musical y Dinámica de la Facultad de Educación de Murcia. Su línea de investigación se centra en la psicodidáctica de la educación física y la motivación hacia la práctica de la actividad física en el contexto escolar.

Juan Antonio Moreno Murcia. Doctor en Psicología. Catedrático de la Universidad Miguel Hernández de Elche donde imparte docencia en el área de Educación física y Deportiva. Sus principales líneas de investigación se centran en el estudio de los factores motivacionales y el análisis de los procesos de enseñanza-aprendizaje en la actividad física y el deporte.

Dirección de los autores: José Antonio Vera Lacárcel

Departamento de Expresión Plástica,

Musical y Dinámica

Facultad de Educación

Universidad de Murcia

Campus de Espinardo, s/n

30100 Murcia

E-mail: verala@um.es

Juan Antonio Moreno Murcia

Universidad Miguel Hernández de Elche

Centro de Investigación del Deporte

Avenida de la Universidad, s/n

03202 Elche (Alicante)

E-mail: J.moreno@umh.es

Fecha Recepción del Artículo: 7. Enero. 2013

Fecha Modificación Artículo: 14. Junio. 2013

Fecha Aceptación del Artículo: 28. Agosto. 2013

Fecha Revisión para publicación: 15. Febrero. 2016 
